\title{
Gating of Acoustic Transducer Channels Is Shaped by Biomechanical Filter Processes
}

\author{
Jennifer Hummel, ${ }^{1}$ Stefan Schöneich, ${ }^{2}$ Manfred Kössl, ${ }^{1}$ Jan Scherberich, ${ }^{1}$ Berthold Hedwig, ${ }^{2}$ Simone Prinz, ${ }^{3}$ \\ and Manuela Nowotny ${ }^{1}$ \\ ${ }^{1}$ Institute of Cell Biology and Neuroscience, Goethe University, 60438 Frankfurt am Main, Germany, ${ }^{2}$ Department of Zoology, University of Cambridge, CB2 \\ 3EJ Cambridge, United Kingdom, and ${ }^{3}$ Max-Planck-Institute for Biophysics, 60438 Frankfurt am Main, Germany
}

Mechanoelectrical transduction of acoustic signals is the fundamental process for hearing in all ears across the animal kingdom. Here, we performed in vivo laser-vibrometric and electrophysiological measurements at the transduction site in an insect ear (Mecopoda elongata) to relate the biomechanical tonotopy along the hearing organ to the frequency tuning of the corresponding sensory cells. Our mechanical and electrophysiological map revealed a biomechanical filter process that considerably sharpens the neuronal response. We demonstrate that the channel gating, which acts on chordotonal stretch receptor neurons, is based on a mechanical directionality of the sound-induced motion. Further, anatomical studies of the transduction site support our finding of a stimulus-relevant tilt. In conclusion, we were able to show, in an insect ear, that directionality of channel gating considerably sharpens the neuronal frequency selectivity at the peripheral level and have identified a mechanism that enhances frequency discrimination in tonotopically organized ears.

Key words: acoustic signal transduction; frequency tuning; insects; transduction channel

\section{Significance Statement}

The evolutionarily conserved ability of sensory cells to sense sound-induced mechanical forces is a fundamental process that still need investigating. In ears, the transduction process of acoustic signals from sound to frequency-specific neuronal responses of sensory cells is based on the opening of mechanosensitive ion channels. Here, we investigated mechanotransduction in the katydid's hearing organ with in vivo measurements of the sound-induced mechanical stimulus and of the electrical responses of the sensory cell at the transduction site. By combining anatomical, biophysical, and neurophysiological data, we present for the first time evidence of a crucial frequency-filter mechanism integral to the channel gating process. This filter takes effect at the first step of the signal transduction chain and shapes behavior-relevant hearing information.

\section{Introduction}

Ears detect sound through the conversion of sound-induced motion into sensory receptor potentials via mechano-electrical transduction of stretch-sensitive ion channels (Garcia-Anoveros and Corey, 1997; Albert et al., 2007; Fettiplace and Kim, 2014). The sensory cells in vertebrate and invertebrate ears have the same evolutionary origin (Burighel et al., 2011; Fritzsch and

Received 0ct. 29, 2015; revised Jan. 7, 2016; accepted Jan. 8, 2016.

Author contributions: M.K. and M.N. designed research; J.H., S.S., J.S., S.P., and M.N. performed research; J.H. analyzed data; J.H., S.S., M.K., B.H., and M.N. wrote the paper.

This work was supported by the German Research Council (Grant N0 841/1-2). We thank Steven Abendroth, Marion Basoglu, and Deryck Mills for excellent technical support; Ian Russell for fruitful discussions and critical comments on an earlier draft of this manuscript; and Scientific and Technical English Language Services (www. stels-ol.de) for help with English.

The authors declare no competing financial interests.

S. Schöneich's present address: Leipzig University, Institute for Biology, Talstra ße 33, D-04103 Leipzig, Germany.

Correspondence should be addressed to Manuela Nowotny, Goethe University, Institute of Cell Biology and Neuroscience, Max-von-Laue-Straße 13, 60438 Frankfurt am Main, Germany. E-mail: nowotny@bio.uni-frankfurt.de.

DOI:10.1523/JNEUROSCI.3948-15.2016

Copyright $\odot 2016$ the authors $\quad 0270-6474 / 16 / 362377-06 \$ 15.00 / 0$
Straka, 2014) and their mechanosensitive ion channels, which are critical for the transduction process, are located at the tips of cilia (Yack, 2004; Peng et al., 2013). The way in which the sensory cells are embedded in the respective ears can be very different and is in each case adapted to the auditory frequency range of interest to each species. In insects, auditory afferents are part of a scolopidial unit (Fig. 1). Each scolopidium consists of one or two bipolar neurons and several supporting cells, including cap and scolopale cells (Yack, 2004). Previous studies using simultaneous recordings in the soma of the sensory neuron and the attached cap cell (Oldfield and Hill, 1986) showed that extracellular compensation currents enable the spike responses of the sensory neuron to also be recordable as passive membrane potential changes in the corresponding cap cell (Fig. $1 B$ ).

Two steps are important for sound processing: (1) the biomechanical transformation of sound waves into a mechanical force that acts on the ciliated structure of the sensory neuron and (2) the subsequent opening of mechanosensitive ion channels that change the neuron's membrane potential by triggering an ion 
A hearing organ
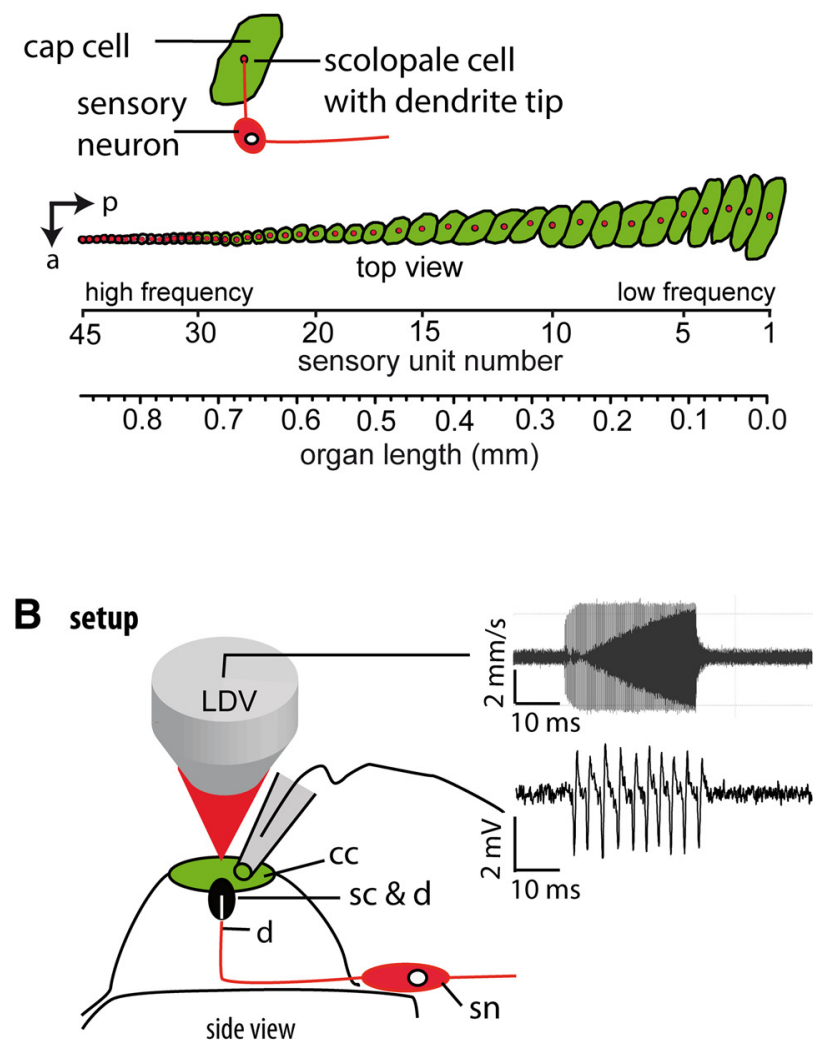

C

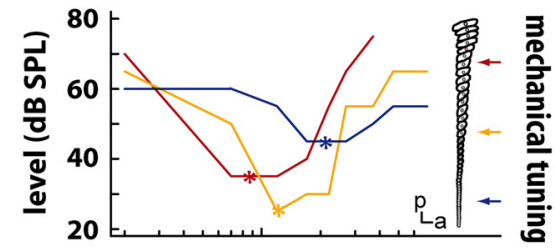

D

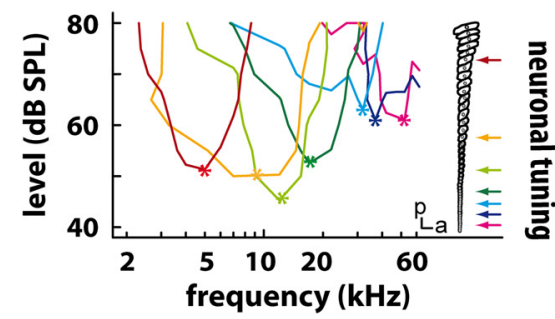

E
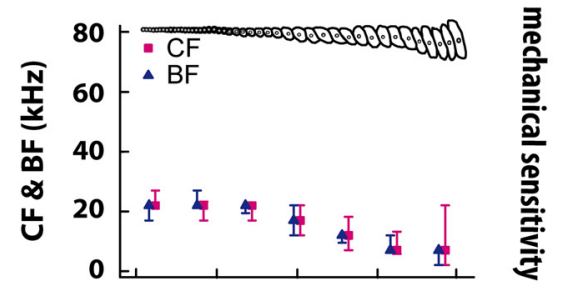

$\mathbf{F}$

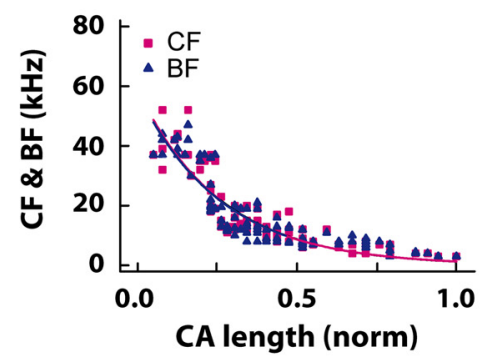

Figure 1. Mechanical and neuronal response measurements in the katydid hearing organ (crista acustica, $C A$ ). $A$, Top view of cellular elements involved in signal transduction along the $C A$, with an indication of organ length and number of sensory units, called scolopidia $(n=13)$. a, anterior; p, posterior. $\boldsymbol{B}$, Measurement setup for the mechanical and neuronal studies shown in a schematic cross-section of the CA. cc, cap cell; $d$, ciliated dendrite; sc, scolopale cell; sn, sensory neuron. Examples of mechanical $(\boldsymbol{C})$ and neuronal ( $\boldsymbol{D}$ ) frequency-tuning curves measured at different positions along the $C A(C: n=1 ; \mathbf{D}: n=5)$. Color-coded arrows along the drawing of the crista acustica on the right mark the exact position of the measurements. A star indicates the $C F$ of each tuning curve. $E$, Median frequency-dependent distribution of CFs and BFs of the mechanical tuning curves at their corresponding position along the $C A(n=10)$. Note that mechanical CFs and BFs are restricted to frequencies $\leq 22 \mathrm{kHz}$. F, Exponential distribution of CFs and BFs of neuronal tuning curves ( $n=106 \mathrm{in} N=32$ animals) at their corresponding position along the organ (trend line: exp. decay, $\left.r^{2}=0.81\right)$. Scale bar, $50 \mu \mathrm{m}$. a, anterior; freq, frequency; p, posterior.

influx. In vertebrates (Dallos and Corey, 1991; Hudspeth et al., 2000; Fettiplace, 2006) and invertebrates (Kamikouchi et al., 2010; Warren et al., 2010; Mhatre and Robert, 2013), both steps of sound processing are subject to amplification and filter mechanisms shaping the sound-induced frequency tuning. Whereas the first filter, represented by a morphology-based tonotopy, has been studied in detail (von Békésy, 1960), filter mechanisms of the second step are still under investigation (Lamb and Chadwick, 2014; Ramamoorthy et al., 2014). Similar to the basilar membrane in the mammalian ear (Russell and Nilsen, 1997; Ren., 2002), sound-induced traveling waves start at the stiff, high-frequency end of the katydid ear and propagate toward more compliant ear structures (Palghat Udayashankar et al., 2012, 2014). These waves are characterized by amplitude and phase components, which indicate the degree and direction of the displacement. This leads to tonotopic frequency processing at a frequency-dependent location where the wave amplitude peaks and the phase response indicates a relatively large delay.

Research on mammalian ears predicts that, for sensitive responses in the high-frequency region of the cochlea, the ampli- tude of sound-induced oscillation of the basilar membrane corresponds directly with the change of electrical current measured in the inner hair cells (Russell et al., 1995; Robles and Ruggero, 2001). This unfiltered gating of mechanical into neuronal responses by the opening of mechanosensitive channels would suggest that the bandwidth and the most sensitive frequency of mechanical and of neuronal tuning match, as is the case in mammals (Narayan et al., 1998). We tested this relationship and investigated the mechano-electrical transduction process in the katydid hearing organ by in vivo measurements of the soundinduced vibration of the organ and by intracellular recordings from its sensory cells. In contrast to the situation in mammals, our findings reveal a broadly tuned mechanical response of the scolopidial organ compared with a sharply tuned neural response of the corresponding sensory cells, which contradicts the idea of unfiltered gating in the insect ear. We argue that the appearance of sensitive cell responses depend on distinct spatial phase changes in the mechanical motion inducing a tilt of the scolopidia. This tilt-dependent neuronal response implies a distinct position of the mechano-electrical transduction channel at the 
dendritic tip, which further suggests that the sensory cells are directionally sensitive.

\section{Materials and Methods}

Animals and preparation. Experiments were performed with adult male and female bushcrickets of the tropical Southeast Asian species Mecopoda elongata L. (Insecta, Orthoptera, Tettigoniidae, $N=65$ ) taken from our own breeding colony at the Department of Cell Biology and Neuroscience in Frankfurt am Main, Germany. We used the dissection technique previously described in detail (Palghat Udayashankar et al., 2012; Hummel et al., 2011). Briefly, the ipsilateral tibia was placed and fixed in a bath chamber filled with saline and the cuticle above the tibial organ was removed.

Histology. For histological studies of hearing organ structures $(n=14)$, tibial parts were subsequently perfused with a fixative of $2 \%$ glutaraldehyde in $0.08 \mathrm{~mol} / \mathrm{L}$ cacodylat buffer and $10 \%$ sucrose and stored overnight at $4^{\circ} \mathrm{C}$ in the same fixative containing 20\% DMSO. Afterward, the tibial parts were washed with cacodylat buffer and dehydrated in alcohols of increasing concentration. In a last step, the organs were incubated stepwise in propylenoxid-Araldite mixtures and finally embedded in pure Araldite. The Araldite blocks were cut into 4- $\mu \mathrm{m}$-thick crosssections using an ultramicrotome (Reichert-Jung Ultracut S; Leica) with glass knifes (Knife-maker, EM KMR2; Leica). For transmission electron microscopy (Tecnai Spirit Biotwin, camera: Gatan US4000), selected cross-sections were transferred to new Araldite blocks for ultrathin sectioning $(\sim 50 \mathrm{~nm})$. Placed on grids, ultrathin sections were contrasted in $5 \%$ uranyl acetate and lead citrate, followed by additional washing steps and final drying.

Laser Doppler vibrometry. Sound-induced crista acustica vibration patterns $(n=21)$ were measured by a microscanning Laser-DopplerVibrometer system (MSV-300 with a sensor head OFV-534; Polytec) attached to a standard microscope (Axio Examiner; Zeiss). The laser beam was positioned under video control (VCT-101; Polytec). For acoustic stimulation, pure tones over a wide range of frequencies (2-77 $\mathrm{kHz}$ in $5-10 \mathrm{kHz}$ steps) were produced by a function generator (NI 611x; Polytec) and fed into a custom-made attenuator to produce different sound pressure levels ( $10-80 \mathrm{~dB}$ SPL in $5 \mathrm{~dB}$ steps). Stimuli were subsequently amplified (RB-850; Rotel) and fed to a broad-band speaker (R2904/700000; ScanSpeak) that was placed $25 \mathrm{~cm}$ from and perpendicular to the ipsilateral spiracle. A condenser microphone (MK301; Microtech Gefell; flat response up to $\sim 80 \mathrm{kHz}$ ) was used for calibration.

Recordings of the resulting mechanical vibration pattern were obtained at a sampling rate of $256 \mathrm{kHz}$ and were averaged 20 times per measurement point (varied between 19 and 25). Data were fast-Fourier transformed at a resolution of $62.5 \mathrm{~Hz}$ to obtain the response characteristics of the crista acustica vibration in response to the frequency pattern of the stimuli. At a certain position along the crista acustica, the characteristic frequency (CF) of mechanical tuning was determined as the frequency with the lowest sound pressure level that caused a mechanical response (response threshold) and the best frequency (BF) as the frequency with the highest displacement values (response maximum). Phase values were obtained in relation to the sound stimulus and phasedelay characteristics were analyzed by calculating the phase difference between the proximal, medial, and distal measuring point and the next measuring point distal to the first. The distance between two measuring points was $35 \mu \mathrm{m}$, which represents the distance between two scolopidial cells. The resulting phase delay difference for each of the three regions were subsequently averaged $(n=10)$.

Electrophysiology. Intracellular recordings of individual cap cells $(n=$ 106 cells, $n=32$ animals) were made using sharp microelectrodes fashioned by a Sutter electrode puller from standard-walled borosilicate glass capillaries (Harvard Apparatus). Filled with $1.5 \mathrm{~mol} / \mathrm{L} \mathrm{KCl}$, the electrodes had final resistances of 60-100 $\mathrm{M} \Omega$. A silver wire was inserted into the Ringer's solution of the leg chamber as the reference electrode. The recorded cell activity was amplified (DC-amplifier BA-03X; NPI) and digitized for storage on a hard drive.

Pure tone stimuli were generated by a data acquisition board (DAP 5216a board; Microstar Laboratories) and the level modified by a programmable attenuator (PA5, TDT PC1; Tucker-Davis Technology). The signal was then amplified (RB-850; Rotel) and fed to the calibrated loudspeaker (R2904/700000; ScanSpeak; calibration see above). The pure tone stimuli of $60 \mathrm{~ms}$ duration (rise/fall time $=4 \mathrm{~ms}$ ) were produced by custom-made software at a repetition period of $250 \mathrm{~ms}$. For the frequency tuning curves, each frequency level combination $(2-62 \mathrm{kHz}$ in $1-5 \mathrm{kHz}$ steps, $30-80 \mathrm{~dB}$ SPL in $5 \mathrm{~dB}$ steps) was randomly presented 5 times.

Data acquisition and evaluation of neuronal responses were performed using custom-made software in Delphi version 4.4.10.92 and MATLAB version 7.6.0 (The MathWorks). First, data were high-pass filtered $(300 \mathrm{~Hz})$ and then a threshold was set well above the noise level. Therefore, all distinct spikes with amplitudes above that noise level were included in further analysis. In a next step, spike waveform analysis was performed to exclude recording artifacts. The tuning curves of single cells were calculated using a threshold criterion of $30 \%$ of maximal spike activity shown in response to all presented frequency level combinations. From these tuning curves, the frequency at the threshold minimum (CF) and the frequency of highest response activity to all presented stimulus combinations $(\mathrm{BF})$ were established.

Statistics. For pooled data ( $N$ : number of animals; $n$ : number of stimulations), median and interquartile range were used to describe the data. Electrophysiological data were fitted by an exponential decay function with two parameters: $f=a * \exp (-b * x)$. Statistical evaluation was performed by Jump version 7.0 (SAS Institute).

\section{Results}

\section{Discrepancy between mechanical and neuronal tuning}

To compare the frequency tuning of sound-induced mechanical and neural responses, we measured the motion and spike activity of the scolopidia along the katydid hearing organ, the crista acustica, to pure-tone stimuli. We found a significant discrepancy between mechanical and neuronal responses to sound stimuli, with the first being more broadly tuned than the second (Fig. $1 C, D)$. Although sensory cells showed CFs that covered the range up to $\sim 50 \mathrm{kHz}$, the CFs of the mechanical tuning curves were restricted to sound frequencies $<22 \mathrm{kHz}$ (Fig. $1 E, F$ ).

\section{Sensory cells are most sensitive where the traveling wave slope is greatest}

Using the neuronally established CF (e.g., proximal: $4 \mathrm{kHz}$ ) of the tuning curves as the stimulus frequency at the respective hearing organ position (Fig. $2 A$, black arrow), we observed that the sensitivity of the neural activity corresponded to a position on the ascending slope of the wave motion rather than to the peak of the wave (Fig. 2A). This characteristic was observed at each frequency place along the entire length of the hearing organ. Soundinduced motion always started at the distal end of the crista acustica with simple up and down movement of the sensory units and without a distinct phase change (Fig. 2B). During the longitudinal propagation toward the proximal part of the hearing organ, phase delays occurred at frequency-specific positions (low stimulus frequencies = proximal part, high stimulus frequencies $=$ distal part) and ended with a sudden phase reversal referred to as the kink (Fig. 2B). Proximal to the amplitude peak, scolopidia moved together in phase without the systematic position phase dependency of the scolopidia distal to the peak (Fig. $2 B$, middle and right).

\section{Neural sensory cell responses depends on direction-sensitive channel gating}

Above, we noted a discrepancy between neuronal and mechanical tuning. By comparing mechanical and neuronal tuning of the same sensory unit along the crista acustica (Fig. 1B), we found that displacement amplitudes of the crista acustica did not always lead to stimulus-induced spike activity of sensory cells (Fig. 2C). 

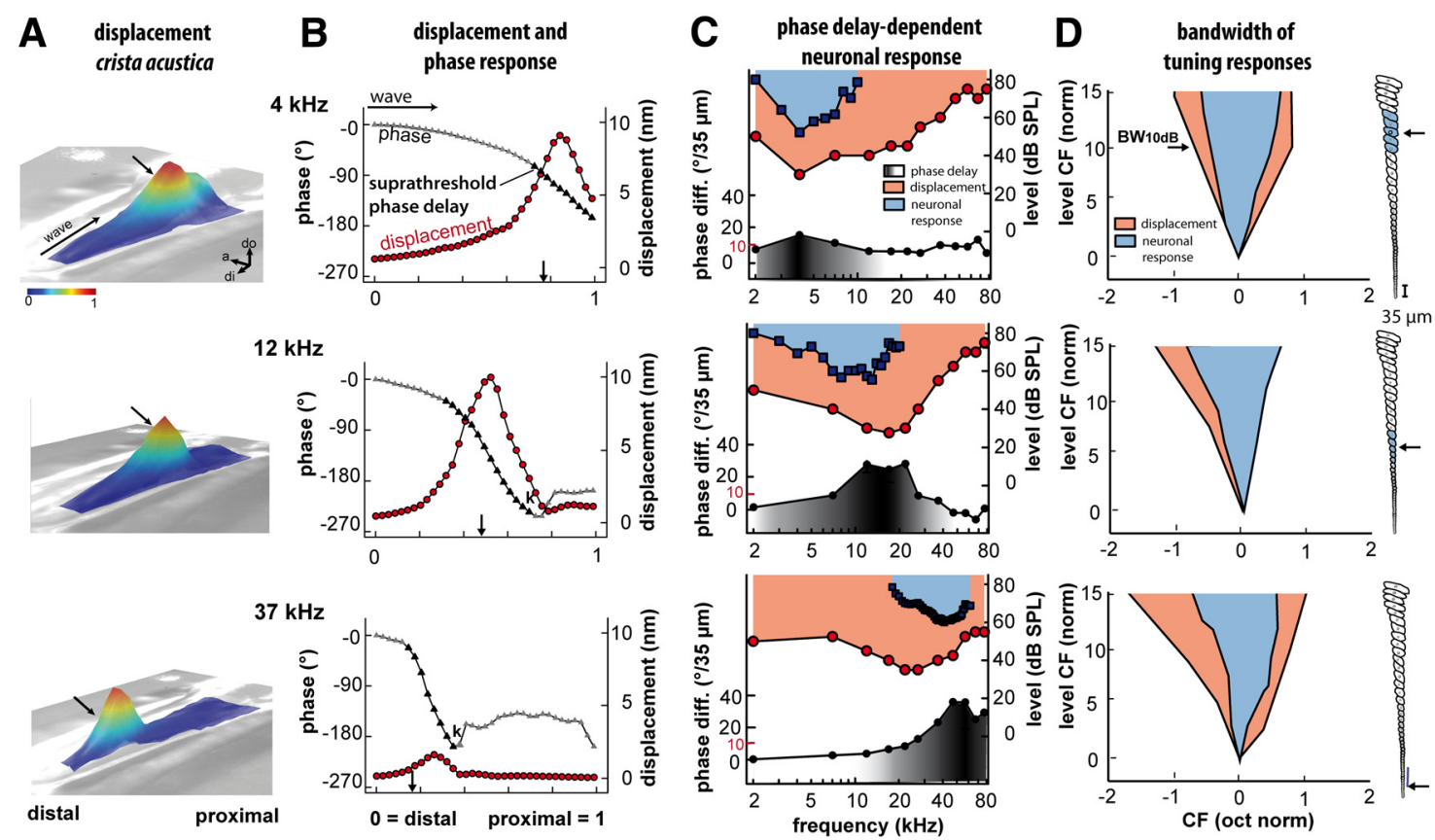

Figure 2. Comparison of mechanical and neuronal tuning of the crista acustica (CA). $A$, Examples (T183) of normalized sound-induced waves that travel along the crista acustica starting at the distal end (stimulus level $80 \mathrm{dBSPL}$ ). a, anterior; di, distal; do, dorsal. $\boldsymbol{B}$, Absolute displacement and phase values along the CA (T138). The phase difference $>10^{\circ} / 35 \mu \mathrm{m}$ between two measurement points (distance $35 \mu \mathrm{m}$ ) is marked by black triangles and $<10^{\circ} / 35 \mu \mathrm{m}$ by gray triangles. A sudden phase change, kink (k), occurs at the end of each wave's amplitude maximum. Black arrows in $A$ and $B$ indicate the position of the sensory units that show the most sensitive neuronal responses to the respective sound-induced mechanical stimulus (top: $4 \mathrm{kHz}$, middle: $12 \mathrm{kHz}$, bottom: $37 \mathrm{kHz}$ ). C, Phase-delay-dependent spike activity of sensory neurons. Phase delays (black circles, left axis, $n=10$ ) at $80 \mathrm{~dB}$ SPL were analyzed at a proximal (top), medial (middle), and distal (bottom) measurement position as indicated in the schematic drawing on the right (black arrows). Phase difference $>10^{\circ} / 35 \mu \mathrm{m}$ correlates with neuronal tuning (blue squares; proximal: $n=9 ;$; medial: $n=$ 20; distal: $n=7$ ) of sensory cells (blue cells in the schematic drawing). Mechanical tuning is shown by red circles. $\boldsymbol{D}$, Comparison of normalized mechanical and neuronal tuning curves as used in $\boldsymbol{C}$. Mechanical tuning (red area; proximal: $n=10$; medial: $n=10$; distal: $n=10$ ) along the CA motion (measurement positions indicated by black arrows) was more broadly tuned than the neuronal tuning (blue area), indicating a biomechanical filter process within the mechano-electrical transduction.

Further, neuronal tuning curves based on spike activity showed similar bandwidths $\left(\mathrm{BW}_{10 \mathrm{~dB}}: 0.9-1.1 \mathrm{~dB} /\right.$ oct; $\left.p>0.63\right)$ at all measured positions along the crista acustica (Fig. 2D). The widths of mechanical tuning curves, however, increased significantly $(p<0.05)$ from the proximal $\left(\mathrm{BW}_{10 \mathrm{~dB}}: 1.1 \mathrm{~dB} / \mathrm{oct}\right)$ to the distal end ( $\left.\mathrm{BW}_{10 \mathrm{~dB}}: 1.9 \mathrm{~dB} / \mathrm{oct}\right)$. Furthermore, we used the phase information of the sound-induced motion to investigate the relationship between mechanical and neuronal responses. A phase delay between two positions along the hearing organ indicates that these two points move at different times in the same direction. By calculating the phase difference (delay) between the recorded position and the next mechanical scanning point (distance between points: $35 \mu \mathrm{m}$ ), we are able to demonstrate that only sounds with a phase difference $>10^{\circ} / 35 \mu \mathrm{m}$ between these two points elicited a neuronal response (Fig. $2 C$ ). The maximum of the motion's phase delay between two measurement points along the crista acustica decreased from $\sim 40^{\circ} / 35 \mu \mathrm{m}$ at the distal end to $17^{\circ} / 35 \mu \mathrm{m}$ at the proximal end. The set threshold of $10^{\circ} / 35 \mu \mathrm{m}$ phase delay between two positions along the hearing organ represents the minimum delay that evoked a direction-sensitive response by a stimulus-relevant tilt between these points along the crista acustica that is able to gate the transduction channels. Here, we resolve the discrepancy between neuronal and mechanical responses by observing that neuronal tuning is tied to the phase of the mechanical response. For a given stimulus frequency range, the region with peak neuronal response corresponds to the region in which the phase of the mechanical response varies most rapidly.

\section{Anatomical data further support finding of a stimulus-relevant tilt}

In M. elongata, sensory cells of the crista acustica feature a single dendrite with a ciliary component at the distal end (Fig. $3 A$ ). The cilium is interrupted by a dilation at the more distal part and ends within the cap, which in turn has a cap cell on top of it. At its basal end, the cilium is stabilized by rootlets. Transmission electron microscopy analysis at five positions along the dendrite revealed a canonical $9 \times 2+0$ microtubule arrangement (Fig. $3 A, B$ ). In contrast to the other positions along the dendrite, we observed additional microtubules at the level of the scolopidial cap surrounding the ciliary tip (Fig. 3B1). Together with the finding that sensory cell responses are only evoked by a distinct phase delay in the mechanical motion of the organ, the anatomical tilt might support the channel-open probability during mechano-electrical transduction (Fig. $3 C$ ).

\section{Discussion}

For hearing to happen, the frequency components of sound signals arriving at the ear must be coded in electrical responses of the auditory afferents by conversion of the stimulus force into the opening of mechanosensitive ion channels. Our results revealed a significant difference between mechanical tuning of crista acustica motion and neuronal tuning of sensory cells. These differences in the bandwidth of mechanical and neuronal tuning curves provide evidence for a biomechanical filter mechanism during mechano-electrical signal transduction that shapes the molecular gating of acoustic transducer channels. Here, we show, in an insect hearing model, that spike activity depends on a dis- 
A

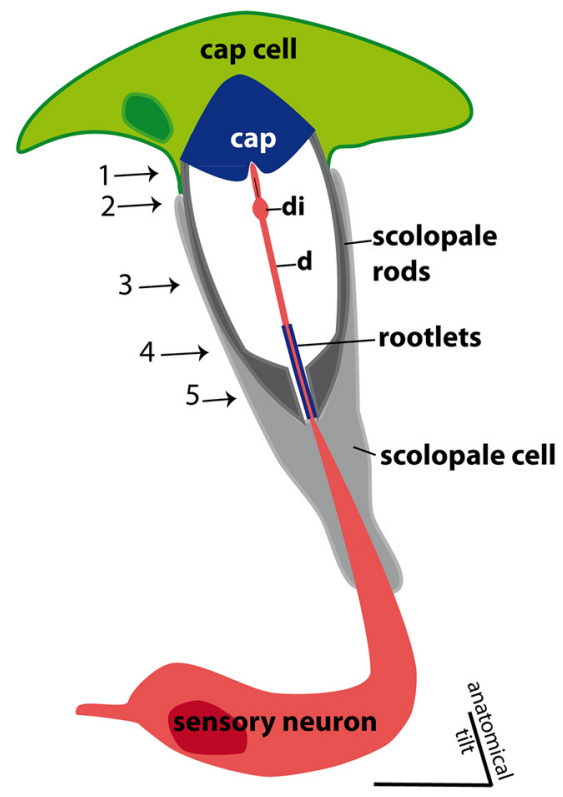

C phase delay

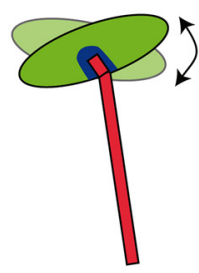

tilt channel open

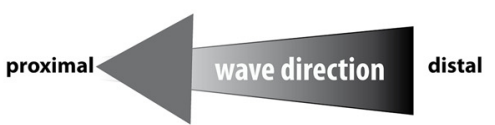

B
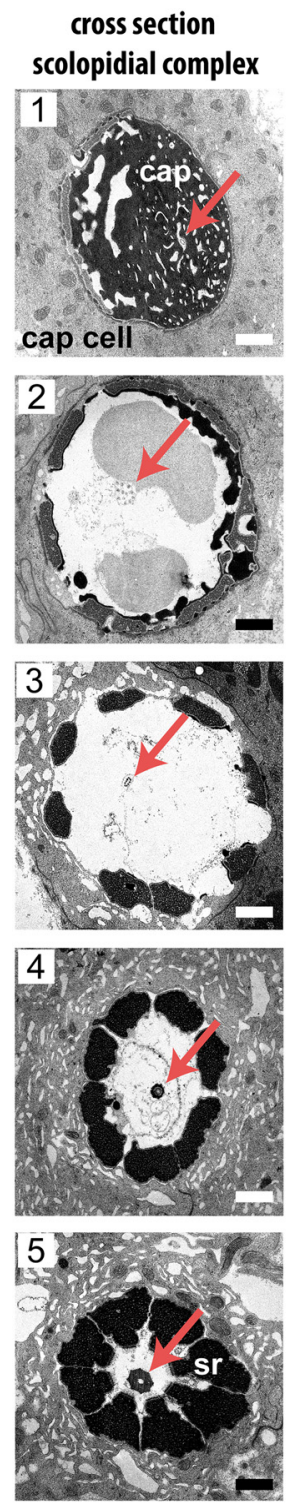

Figure 3. Mechano-electrical excitation of the afferent dendrite. $A$, Microanatomy of the scolopidial unit in a longitudinal cross-section. di, dilation of the sensory dendrite; $d$, dendrite. $\boldsymbol{B}$, At five different levels, the dendrite tip in the lumen of the scolopale cell was transected $(n=1)$. Transmission electron microscopy images depict the arrangement of the scolopale cell and inner structure of the ciliated sensory dendrite (red arrows). The right row of images shows the dendrite cross-section at a higher magnification. Additional microtubules are present in the ciliated tip of the dendrite covered by the cap cell (yellow arrow in right panel 1). Scale bar, $1 \mu \mathrm{m}$ left row and $0.1 \mu \mathrm{m}$ right row. C, Pictogram illustrates the mechanism of force transduction by a phase-change-induced tilt that opens mechanosensitive ion channels.

tinct phase delay in the mechanical motion, which induces a tilt of the organ. This is consistent with the fact that we found the optimal position of the sensory unit that elicits sensitive responses to be on the ascending slope of the wave. Therefore, a tilt of the organ increases the channel-open probability, whereas channels are closed when structures simply move up and down in synchrony.

Typically for the kinocilium-based transduction apparatus found in all taxa along the metazoa clade (Fritzsch and Straka, 2014), the cross-section of the sensory dendrite of katydids shows a canonical $9 \times 2+0$ microtubule arrangement. Our preliminary anatomical investigations provide evidence for microtubule-like structures surrounding the very tip of the cilium. These microtubules might anchor filaments relevant to gating. Similar structures have been found in the tips of
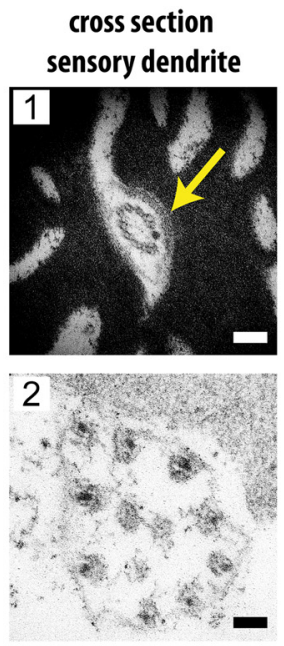

3

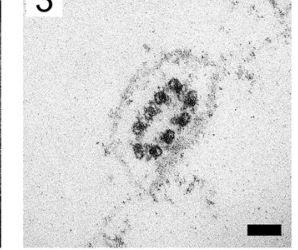

4
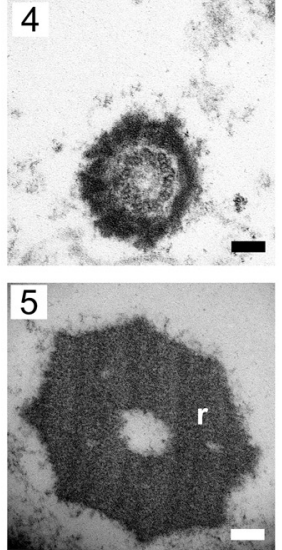

campaniform sensilla in Drosophila (Liang et al., 2013). In these animals, compliant filamentous structures connect the plasma membrane to such microtubules, indicating that they act as gating springs on mechanotransduction channels that are located in the tip of these organs (Lee et al., 2010; Kittelmann and Göpfert, 2014). In vertebrate hair cells, gating springs are also located at the tip of hair cell stereocilia. Here, channels open when negative deflection toward the taller stereocilia stretches transduction links (Hudspeth et al., 2000). In contrast, a positive deflection of stereocilia toward the shorter stereocilia results in a relaxation of transduction links and thus channels are closed. The same principle is true for the scolopidia in katydids. Channels open by a distinct tilt of the sensory epithelium in the proximal direction that is induced by the traveling wave. Conversely, channels are closed when the scolopidia are deflected toward the distal direction, which happens when the traveling wave collapses and therefore the phase of the mechanical vibration is reversed (kink), indicating a tilt of the sensory epithelium in this opposite direction. Inferred from mechanisms existing in mammals (Hudspeth et al., 2000) and flies (Liang et al., 2013), the sound-induced tilt of the sensory epithelium is most likely accompanied by a distinct tilt of single scolopidial cells at the tip of the dendrites as well. Therefore, the characteristics of sensory cell excitation and the finding of microtubules surrounding the cilium suggest the occurrence of gating springs at the transduction site.

Assuming that the transduction channel is located at the ciliated tip of the sensory dendrite (Liang et al., 2013), an anatomical tilt of the transduction apparatus increases the force acting on the transduction channel. We and others (Oldfield, 1982; Lin et al., 1993) observed an anatomical tilt of the transduction apparatus in the proximal direction. Therefore, we presume that this anatomical tilt could induce the acting force and thereby further increase a phasedependent channel-opening probability. This is supported by a recent cochlear model (Lamb and Chadwick, 2014) that points to the importance of an angle between structures involved in the transduction process for providing a band-pass filter, which serves as an input to a nonlinear force in the direction of channel gating.

Due to technical limitations, the mechano-electrical transduction process is poorly understood both in vertebrates and invertebrates. In the katydid ear, we are able to overcome these limitations and found that a pronounced phase delay is critical for the opening of stretch-sensitive transduction channels in the ciliated tip of the sensory neurons in these hearing organs 
by providing a tilt between dendrite tip and the overlying cap cell. Our data show that the distribution of distinct phase changes between two measurement points corresponds to neuronal tuning, which indicates that mechanosensitive transduction channels in the dendrite are only activated by displacement amplitudes that are accompanied by a certain phase delay, so a longitudinal differential motion of the crista acustica. In contrast, neuronal responses in mammals even correspond to a nontraveling wave mode of basilar membrane motion (Huang and Olson, 2011). However, basilar membrane motion that excites sensory cells that are tuned to frequencies above the CF has to be larger by a factor of 10 than the motion that is excitatory at the $\mathrm{CF}$, suggesting that longitudinal differential motion helps in increasing the sensitivity in mammals as well.

In conclusion, directionality of the channel-gating process provides a biomechanical second filter in bushcrickets that shapes the frequency range and sharpens the frequency tuning of sensory neurons. Moreover, neuronal tuning expands the dynamic range of frequency hearing, because neuronal tuning curves show a larger range of CFs than their corresponding mechanical tuning curves. Therefore, from the outset of the hearing process, the characteristics of the coupling between mechanical stimulus and sensory cell excitation in bushcrickets significantly affect the animal's auditory perception.

\section{References}

Albert JT, Nadrowski B, Göpfert MC (2007) Drosophila mechanotransduction: linking proteins and functions. Fly (Austin) 1:238-241. CrossRef Medline

Burighel P, Caicci F, Manni L (2011) Hair cells in non-vertebrate models: lower chordates and molluscs. Hear Res 273:14-24. CrossRef Medline

Dallos P, Corey ME (1991) The role of outer hair cell motility in cochlear tuning. Curr Opin Neurobiol 1:215-220. CrossRef Medline

Fettiplace R (2006) Active hair bundle movements in auditory hair cells. J Physiol 576:29-36. CrossRef Medline

Fettiplace R, Kim KX (2014) The physiology of mechanoelectrical transduction channels in hearing. Physiol Rev 94:951-986. CrossRef Medline

Fritzsch B, Straka H (2014) Evolution of vertebrate mechanosensory hair cells and inner ears: toward identifying stimuli that select mutation driven altered morphologies. J Comp Physiol A Neuroethol Sens Neural Behav Physiol 200:5-18. CrossRef Medline

Garcia-Anoveros J, Corey DP (1997) The molecules of mechanosensation. Annu Rev Neurosci 20:567-594. CrossRef Medline

Huang S, Olson ES (2011) Auditory nerve excitation via a non-traveling wave mode of basilar membrane motion. JARO 12:559-575. CrossRef Medline

Hudspeth AJ, Choe Y, Mehta AD, Martin P (2000) Putting ion channels to work: mechanoelectrical transduction, adaptation, and amplification by hair cells. Proc Natl Acad Sci U S A 97:11765-11772. CrossRef Medline

Hummel J, Kössl M, Nowotny M (2011) Sound-induced tympanal membrane motion in bushcrickets and its relationship to sensory output. J Exp Biol 214:3596-3604. CrossRef Medline

Kamikouchi A, Albert JT, Göpfert MC (2010) Mechanical feedback amplification in Drosophila hearing is independent of synaptic transmission. Eur J Neurosci 31:697-703. CrossRef Medline
Kittelmann M, Göpfert MC (2014) Mechanisms and genes in Drosophila hearing. e-Neuroforum 5:72-76. CrossRef

Lamb JS, Chadwick RS (2014) Phase of shear vibrations within cochlear partition leads to activation of the cochlear amplifier. PLoS One 9:e85969. CrossRef Medline

Lee J, Moon S, Cha Y, Chung YD (2010) Drosophila TRPN (=NOMPC) channel localizes to the distal end of mechanosensory cilia. PLoS One 5:e11012. CrossRef Medline

Liang X, Madrid J, Gärtner R, Verbavatz JM, Schiklenk C, Wilsch-Bräuninger M, Bogdanova A, Stenger F, Voigt A, Howard J (2013) A NOMPCdependent membrane-microtubule connector is a candidate for the gating spring in fly mechanoreceptors. Curr Biol 23:755-763. Medline

Lin Y, Kalmring K, Jatho M, Sickmann T, Rössler W (1993) Auditory receptor organs in the forelegs of Gampsocleis gratiosa (Tettigoniidae): morphology and function of the organs in comparison to the frequency parameters of the conspecific song. J Exp Zool 267:377-388. CrossRef

Mhatre N, Robert D (2013) A tympanal insect ear exploits a critical oscillator for active amplification and tuning. Curr Biol 23:1952-1957. CrossRef Medline

Narayan SS, Temchin AN, Recio A, Ruggero MA (1998) Frequency tuning of basilar membrane and auditory nerve fibers in the same cochleae. Science 282:1882-1884. CrossRef Medline

Oldfield BP (1982) Tonotopic organisation of auditory receptors in Tettigoniidae (Orthoptera: Ensifera). J Comp Physiol A Neuroethol Sens Neural Behav Physiol 147:461-469. CrossRef

Oldfield BP, Hill KG (1986) Functional organization of insect auditory sensilla. J Comp Physiol A Neuroethol Sens Neural Behav Physiol 158:27-34. CrossRef

Palghat Udayashankar A, Kössl M, Nowotny M (2012) Tonotopically arranged traveling waves in the miniature hearing organ of bushcrickets. PLoS One 7:e31008. CrossRef Medline

Palghat Udayashankar A, Kössl M, Nowotny M (2014) Lateralization of travelling wave response in the hearing organ of bushcrickets. PLoS One 9:e86090. CrossRef Medline

Peng AW, Effertz T, Ricci AJ (2013) Adaptation of mammalian auditory hair cell mechanotransduction is independent of calcium entry. Neuron 80:960-972. CrossRef Medline

Ramamoorthy S, Zha D, Chen F, Jacques SL, Wang R, Choudhury N, Nuttall AL, Fridberger A (2014) Filtering of acoustic signals within the hearing organ. J Neurosci 34:9051-9058. CrossRef Medline

Ren T (2002) Longitudinal pattern of basilar membrane vibration in the sensitive cochlea. Proc Natl Acad Sci U S A 99:17101-17106. CrossRef Medline

Robles L, Ruggero MA (2001) Mechanics of the mammalian cochlea. Physiol Rev 81:1305-1352. Medline

Russell IJ, Nilsen KE (1997) The location of the cochlear amplifier: spatial representation of a single tone on the guinea pig basilar membrane. Proc Natl Acad Sci U S A 94:2660-2664. CrossRef Medline

Russell IJ, Kössl M, Murugasu E (1995) A comparison between toneevoked voltage responses of hair cells and basilar membrane displacements recorded in the basal turn of the guinea pig cochlea. In: Advances in Hearing Research (Manley GA, Klump GM, Köppl C, Fastl HG, Oeckinghaus H, eds), pp 136-144. Singapore: World Scientific Publishers.

von Békésy G (1960) Experiments in hearing. New York: McGraw Hill.

Warren B, Lukashkin AN, Russell IJ (2010) The dynein-tubulin motor powers active oscillations and amplification in the hearing organ of the mosquito. Proc R Soc B 277:1761-1769. CrossRef Medline

Yack JE (2004) The structure and function of auditory chordotonal organs in insects. Microsc Res Tech 63:315-337. CrossRef Medline 